\title{
Rietveld texture analysis from diffraction images
}

\section{Lutterotti ${ }^{1, *}$, M. Bortolotti ${ }^{1}$, G. Ischia ${ }^{1}$, I. Lonardelli ${ }^{2}$, H.-R. Wenk ${ }^{2}$}

${ }^{1}$ Department of Materials Engineering and Industrial Technologies, University of Trento, via Mesiano 77, 38050, Italy

${ }^{2}$ Department of Earth and Planetary Science, University of California at Berkeley, CA, USA

* Contact author; e-mail: Luca.Lutterotti@ing.unitn.it

Keywords: Rietveld refinement, texture, image plate, CCD, X-ray diffraction

\begin{abstract}
The procedure to perform Rietveld texture analysis from diffraction images collected with CCDs or image plates is shown. In some cases only one transmission image may be sufficient to obtain an Orientation Distribution Function (ODF) if a sufficient number of peaks are included. The images are transformed in spectra and analyzed using the Maud Rietveld program containing some recently developed texture model well suited for this kind of analysis. In this work we will present the results obtained using a custom laboratory image plate camera developed for texture analysis. The instrument may work with a curve image plate detector in reflection condition or with a flat detector in transmission. The reflection condition is used mainly for ceramics and metal-alloys and the transmission mode for polymers and fibres. We will show how with the combination of such camera and the Rietveld Texture Analysis method we were able to analyze the ODF of the martensitic phase of Shape Memory Alloy (monoclinic NiTi SMA) as well as to obtain the quantitative texture of low symmetry polymers in fibre form.
\end{abstract}

\section{Introduction}

The Rietveld texture analysis is an effective new way to analyse the texture of complex samples in which multiple overlapping phases or low symmetry compounds are present and the traditional pole figures analysis cannot be applied. This type of analysis is well suited also for data collected on instruments with large position sensitive or 2-dimensional detectors, where it is quicker to collect more reflections at few tilting conditions than to obtain few sufficiently covered pole figures. This type of instruments is spreading up recently, mostly on synchrotron and neutron facilities but also in normal labs. We have recently developed the methodology, software and procedures to take advantage of 2-dimensional data coming from CCD and image plate cameras in Rietveld texture analysis and we have shown some applications from biological sample to metal alloys $[1,2]$. We will show here some additional examples for low symmetry alloys and polymers with data collected on a lab instrument. In the polymer field, diffraction images are already used for qualitative orientation analysis. In our 
case we were able to extract quantitative texture data (the Orientation Distribution Function, ODF) as well as to refine the polymer structure.

\section{Theory and methodology}

With 2D detectors the possible geometries can be divided for our purposes in transmission and reflection. In transmission the more traditional setup is the classical Laue camera that can be used with a monochromatic beam and equipped with an image plate instead of the normal film. Such type of instruments is becoming available more and more at synchrotron facilities, in the last few years, and they are very convenient for quick texture analyses. To increase the coverage of the pole figures is sufficient to tilt the sample to an axis laying in the same plane as the 2D detector and few tilting are necessary. For transmission images thin sample are required or highly penetrating X-rays. Another arrangement suitable for thick sample and laboratory instruments uses the $2 \mathrm{D}$ detector in reflection. Figure 1 shows the scheme of a camera equipped with a large curved image plate detector realized in our lab. A typical image collected with such instrument is reported on the right of figure 1 . The partial Laue circles in reflection are shown and typically the $2 \theta$ range covers from 0 to $120^{\circ}$. Few rotations around the $\varphi$ axis on the figure 1 are necessary to cover the ODF for most of the samples.

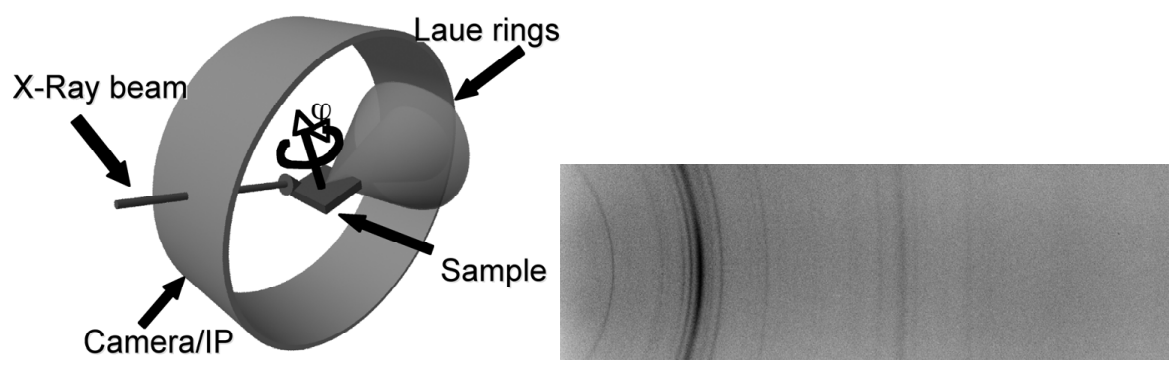

Figure 1. On the left: scheme of the laboratory image plate camera. On the right: an image obtained from the curved image plate in reflection.

The images from both geometries are processed by integrating the intensities along radial slices every $5^{\circ}$ to obtain spectra as in figure 2 . For the curved image plate in reflection the integration goes along ellipsoids and the slices are not following straight lines. Also for the transmission case we choose to use our integration routines implemented in Maud to keep the integration information with the intensity data and transform into $2 \theta$ information only during the Rietveld computation allowing the possibility to optimize the centre, tilting positioning of the images and camera radius if necessary during the refinement. By this procedure we get a better angular calibration and results and we can use it for high-pressure experiments where the Laue circles are no more circles.

The spectra obtained this way can be processed by a Rietveld texture analysis.

The Rietveld Texture Analysis (RTA) has been described elsewhere [6]. Here we will give some details of two texture models developed and included in the program Maud specifically for this kind of analysis. They are EWIMV and Standard Functions. 

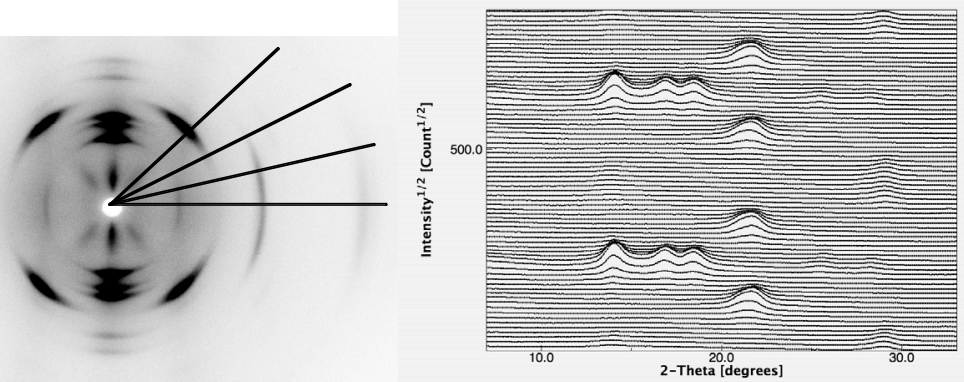

Figure 2. On the left: caking of the image in slices from the centre to obtain the spectra (each every $5^{\circ}$ circularly in this example) on the right ready to be processed in the Rietveld texture package.

EWIMV (derived from WIMV) is part of the discrete texture methods. The ODF space described by the three eulerian angles is divided in small cells each one defining the value of the ODF in its volume. The pole figure value for each peak of each spectrum is computed by a numerical integral throughout the ODF space considering a tube projection or averaging over adjacent cells. In Maud an external cycle performed once every refinement cycle extracts the pole figure values from the spectra using a tuned Le Bail algorithm and computes the ODF through an entropy method. The ODF is used in the subsequent fitting or refinement cycle to compute the texture intensities (pole figure values) for each peak/point. The entropy algorithm ensures the smoothest positive solution for the ODF. A positive ODF is always guaranteed. Another difference respect to WIMV is that the pole figure values are weighted in the algorithm proportionally to the square root of the peak intensity.

In Maud there are two types of standard functions [5]: a fibre component and a spherical component (linear combination of a Gauss and Lorentz standard function).

The Gauss spherical component is defined as:

$$
f(S, \widetilde{\omega})=N(S) e^{S \cos \widetilde{\omega}} \geq 0,0 \leq \widetilde{\omega}=\widetilde{\omega}\left(g_{0}, g\right) \leq \pi, 0 \leq S \leq \infty
$$

where:

$$
N(S)=\left[I_{0}(S)-I_{l}(S)\right]^{-1}, I_{l}(x)=\frac{1}{\pi} \int_{0}^{\pi} e^{x \cos t} \cos (l t) d t
$$

$I_{l}$ is the modified Bessel function. The Lorentz spherical component is instead:

$$
f(t, \widetilde{\omega})=\left(1-t^{2}\right) \frac{\left(1+t^{2}\right)^{2}+4 t^{2} \cos ^{2}(\widetilde{\omega} / 2)}{\left(1+t^{2}\right)^{2}-4 t^{2}\left[\cos ^{2}(\widetilde{\omega} / 2)\right]^{2}}>0, t<1
$$

$\mathrm{g}_{0}$ and $\mathrm{g}$ are the eulerian coordinates in the ODF space, the first being the position of the maximum of the spherical component. A fibre component is obtained integrating a Gauss spherical component around the fibre axis $\mathrm{n}$ :

$$
f\left(S, \bar{n}, g_{0}, g\right)=N(S) I_{0}\left(S \frac{1+z}{2}\right) e^{S(z-l) / 2}, z=\left(\bar{n} \cdot g_{0}^{-1} \cdot g \cdot \bar{n}\right)
$$

The advantages of the standard functions for texture in the Rietveld refinement are that the texture is described by refinable parameters, very sharp textures can be modelled, can be constrained to be always positive, very smooth ODF easy to understand are obtained with few parameters. The disadvantages are that they require a definition of starting components 
(so often a first analysis using another method is necessary) and that complex textures require several components.

\section{Analysis examples and discussion}

The first successful example is the comparative texture analysis of a dinosaur tendon and a salmon scale. The data was collected in transmission with a MAR image plate at the highenergy beam line 11-ID at APS for the tendon and with the CCD of ID13 at ESRF for the salmon scale. The full analysis and discussion is reported in [1] and for brevity we will not repeat here. This first analysis has showed the feasibility of the method and the good results obtainable even if a micro beam was employed and a very poorly crystallized sample was analyzed like for the salmon scale. The second example is the texture analysis at high temperature of the Ti phase transition. In this case the sample was analyzed also in transmission and for technical constraint no tilting of the sample was possible and the texture analysis was conducted on only one image. The full details are available in [2] where the successful texture analysis is presented.
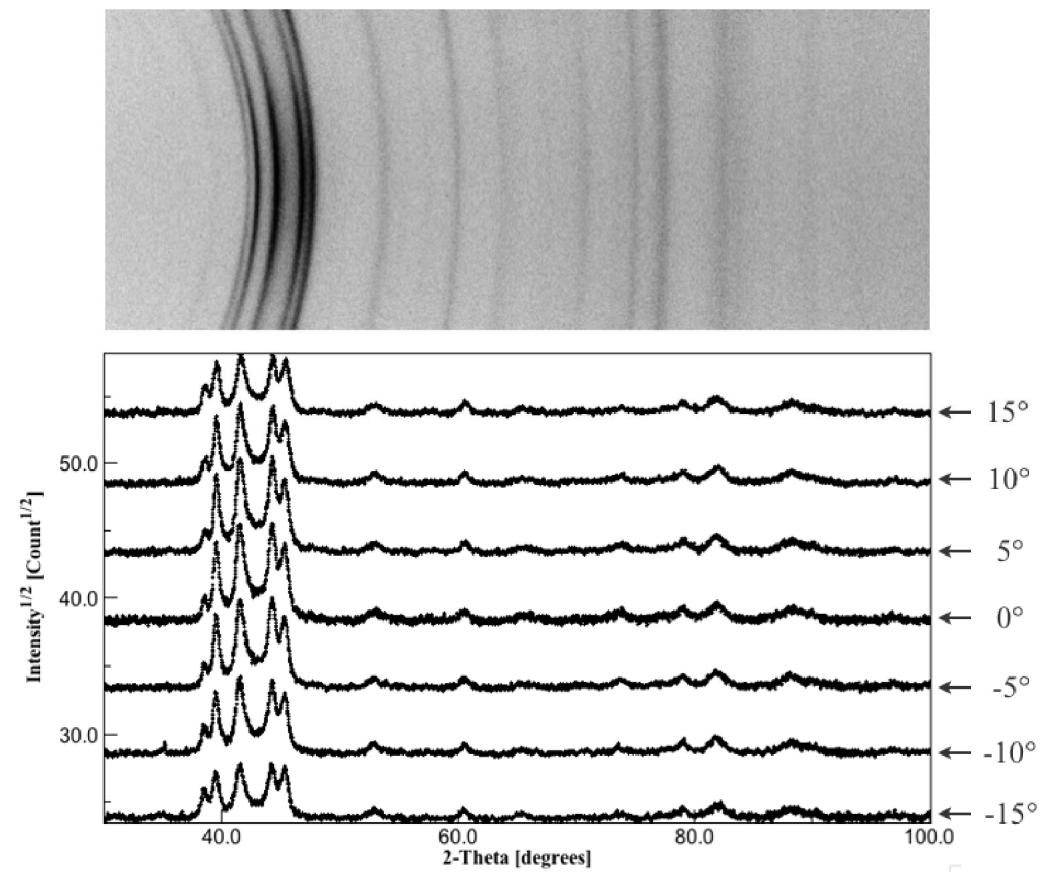

Figure 3. One of the diffraction images (top) collected for the NiTi sample with the lab image plate reflection camera. The partial Laue circles are transformed by integration in spectra and fitted in Maud to obtain the texture, quantitative phase analysis and structural information (bottom). 

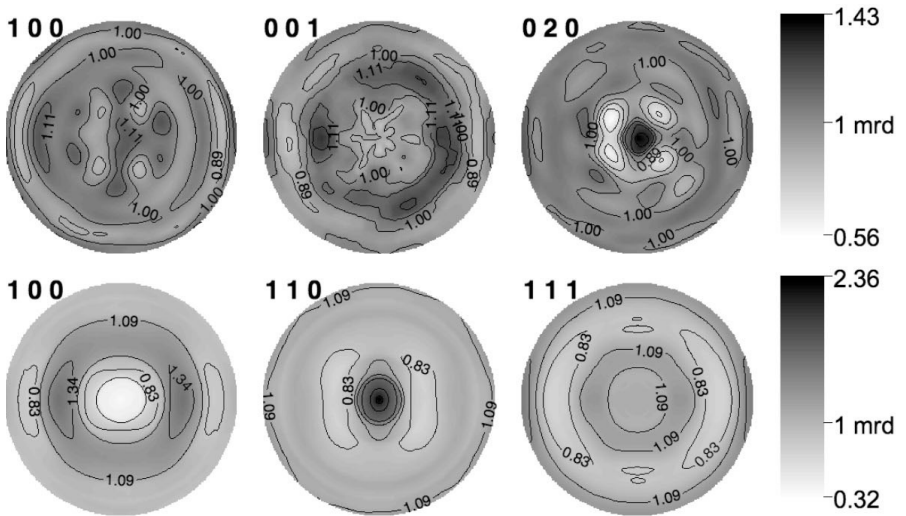

2.36

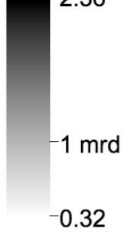

Figure 4. Reconstructed pole figures from ODF obtained by the Rietveld texture analysis for the monoclinic NiTi phase (top) and the cubic NiTi phase (bottom).

The last two examples were conducted using in lab instruments equipped with image plate detectors. The first one is a texture analysis of the martensitic-austenitic phase transformation of a NiTi shape memory alloy (SMA). For the monoclinic phase, due to the high peak overlapping, a complete quantitative texture analysis of this kind of structure has never been done (only for the cubic, austenitic phase). We employed successfully the laboratory image plate camera in reflection described earlier (texture variant of the Italstructures IPD 3000), using 6 rotations for $\varphi$ (each every $60^{\circ}$ ) corresponding to 6 images and 3 omega positions. Figure 3 (top) shows one of these images were the texture variation of the intensity along the circles is clearly visible. On the bottom some spectra are reported (only $\pm 15^{\circ}$ along the circles as they are the only going through the entire image plate) with the fitting. The reconstructed pole figures from the ODF at the end of the analysis using EWIMV are reported in figure 4 for the low temperature monoclinic martensite structure and the high temperature austenitic cubic phase. The work is in progress to characterize the variant selection rules for this transformation. The last example is an isotactic polypropylene fibres sample analysed in transmission.

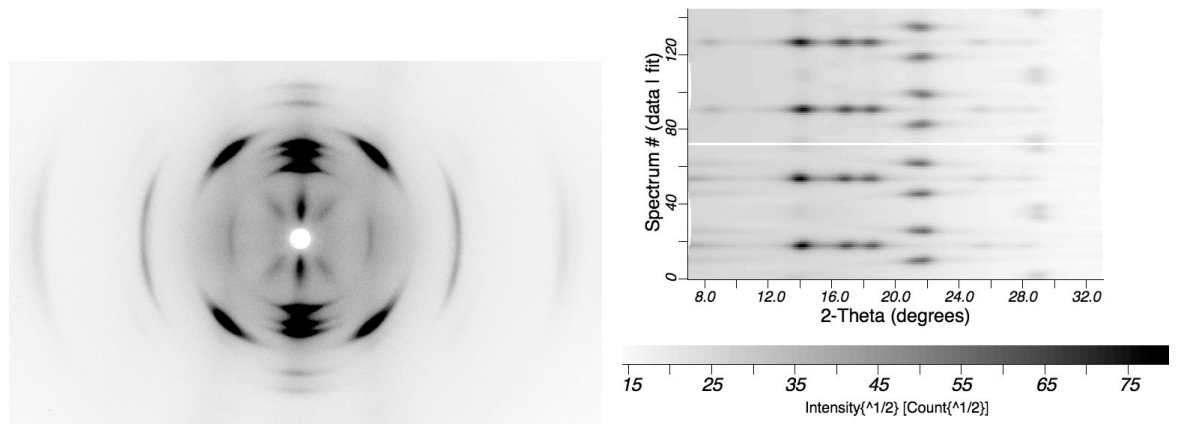

Figure 5. On the left: Laue transmission image of the polypropylene fibres. The fibres were aligned horizontally. On the right: experimental (bottom) and refined (top) spectra. 


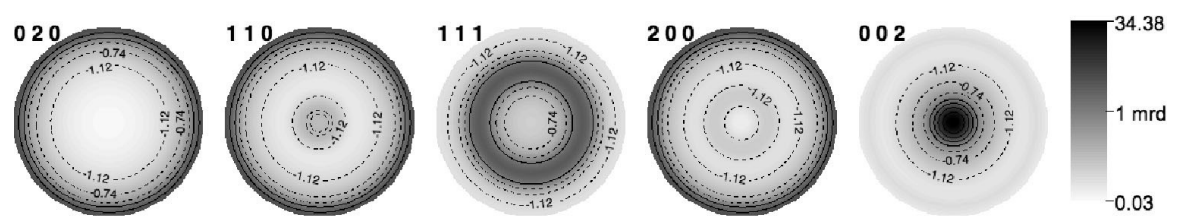

Figure 6. Equal area reconstructed pole figures for polypropylene from the ODF obtained by Rietveld texture analysis using the standard function texture model. The mean spread of the fibres is $15.7^{\circ}$ from the fitting. Intensity in logarithmic scale.

The diffraction image collected with the lab instrument and the Rietveld fitting is shown in figure 5. Figure 6 reports the texture results. The standard functions texture model has been used and a mean fibre texture spreading of $15.7^{\circ}$ from the principal axis has been found. The crystal structure of the polypropylene was refined successfully starting from the proposed structure of Natta and co-workers and was found significantly different [4].

\section{Concluding remarks}

Using the Rietveld texture analysis in combination with data from 2-dimensional detectors is proving a reliable and fast way to analyse quantitatively complex samples. The information obtained is not limited to the ODF, but structural and microstructural information are obtained as well. Care should be used to refine the positional and image tilting errors inside the Rietveld for best results. The new texture models introduced in the Rietveld, EWIMV and standard functions, proved to be well suited for this kind of analysis. In particular the last one can be used also with polymer with low symmetry and crystallization where the peak overlap is quite severe even for a refinement. The strong texture permits a better separation of the different reflections contribution allowing a successful refinement.

\section{References}

1. Lonardelli, I., Wenk, H. -R., Lutterotti, L. \& Goodwin, M., 2005, J. Synchrotron Rad., 12 [3], 354.

2. Ischia, G., Wenk, H. -R., Lutterotti, L. \& Berberich, F., 2005, J. Appl. Cryst., 38, 377.

3. Maud: Materials Analysis Using Diffraction, Version 2.0, 2006, http://www.ing.unitn.it/ maud.

4. Lutterotti L., Bortolotti M. \& Fambri L., 2005, Acta Crystallographica, A61, C391.

5. Matthies, S., Vinel, G.W. \& Helming, K., 1987, Standard Distributions in Texture Analysis (Berlin: Akademie Verlag).

6. Lutterotti, L., Matthies, S., Wenk, H.-R., Schultz, A. S. \& Richardson, J.W.Jr, 1997, J. Appl. Phys., 81, 594.

Acknowledgements. The authors wish to thank the APS and ESRF synchrotron facilities for some of the experiments and Italstructures for the support on building the image plate lab instrumentation. 